\title{
ORIENTAÇÃO DE DIETA PARA REALIZAÇÃO DA ESPECTROFOTOMETRIA DO REFLUXO
}

\author{
Valter Nilton FELIX e Ricardo Guilherme VIEBIG
}

\begin{abstract}
RESUMO - Racional - O probe espectrofotométrico de detecção de refluxo duodenogastroesofágico, utilizando a bilirrubina como marcador, pode sofrer interferência de alimentos de forte coloração, gerando falsas leituras. Objetivo - Verificar a absorbância de 48 alimentos para identificar aqueles que não interferem com os resultados do probe espectrofotométrico. Método - Os alimentos secos foram preparados com água ou leite e os outros triturados ou examinados in natura, no próprio recipiente de calibração do probe. Resultados - A absorbância dos alimentos de cor fraca habitualmente é menor que 0,14 unidade, o limite normal para registros e traçados na pesquisa de refluxo biliar. Dos 48 alimentos testados, 32 demonstraram absorbância menor que o limite estabelecido, mostrando-se adequados, portanto, para serem administrados durante a realização da espectrofotometria. Ao contrário, 16 estão contra-indicados, podendo alterar os resultados do exame: ovo mexido, mingau de farinha láctea, feijão verde, beterraba, cenoura, chuchu, abobrinha, mandioquinha, milho verde cozido, laranja, caju, uva roxa, abacate, manga, mamão e pêssego. Conclusãa - Alimentos com absorbância baixa são capazes de constituir agradável dieta, recomendável no período de exame.
\end{abstract}

DESCRITORES- Refluxo duodenogástrico. Refluxo gastroesofágico. Refluxo biliar. Esofagite péptica. Espectrofotometria. Dieta.

\section{INTRODUÇÃO}

A espectrofotometria do refluxo tem-se mostrado capaz de auxiliar com importância o diagnóstico da doença do refluxo gastroesofagiano (DRGE) $)^{(1,4,5,7)}$.

$\mathrm{O}$ probe de fibra óptica desenvolvido por BECHI et al. $^{(2)}$ (Bilitec $2000^{\circledR}$ - Figura 1) utiliza sabidamente a bilirrubina como marcador. Visa a medir as propriedades absortivas do fluido em relação ao feixe de luz emitido, cujo comprimento de onda é igual ou maior que o da bilirrubina; assim, tem-se maior absorbância, quanto mais bilirrubina está presente no fluido.
Na prática, se o probe é posicionado no esôfago, à semelhança de um eletrodo de pHmetria, e há refluxo biliar patológico, a absorbância registrada pelo probe de espectrofotometria é expressiva ${ }^{(5)}$.

A limitação do método é alimento ou bebida que tenha capacidade de interferir na colorimetria e gerar falsa absorbância de bilirrubina ${ }^{(2)}$, daí a necessidade de se estabelecer dieta adequada ao paciente que esteja no curso de espectrofotometria do refluxo gastroesofagiano (RGE).

Alimentos consistentes, fibrosos ou grãos íntegros também são contra-indicados durante a espectrofotometria do RGE, pois podem aderir ao probe e bloquear a leitura óptica ${ }^{(2)}$.

MODINE - Motilidade Digestiva e Neurogastroenterologia

Endereço para correspondência: Dr. Valter Nilton Felix - Rua Frei Caneca, 1407, cj. 221 - 01307-003 - São Paulo, SP. e-mail: v.felix@ terra.com.br. 


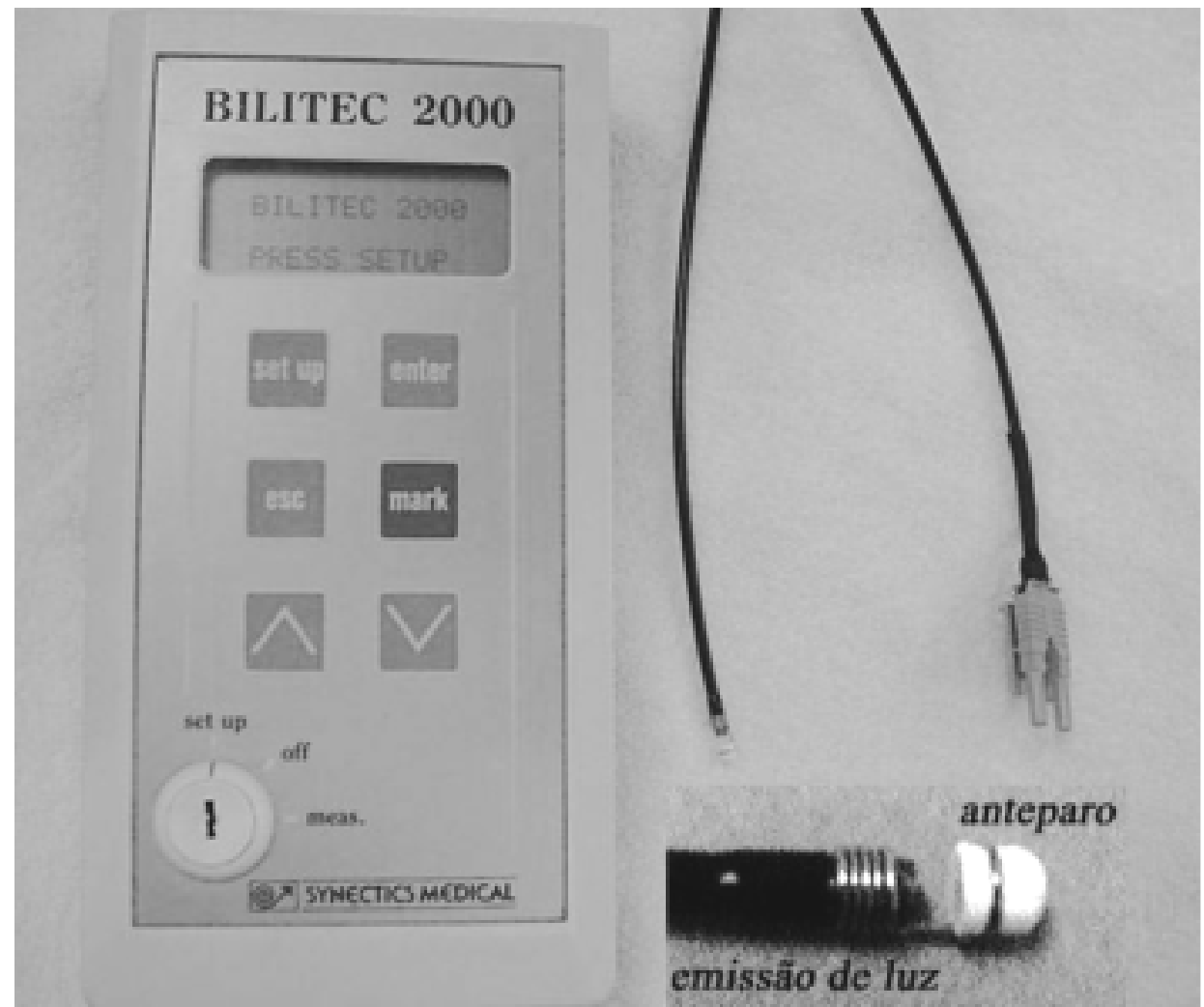

FIGURA 1 - Espectrofotômetro portátil e detalhes do probe.

O objetivo deste estudo foi listar alimentos que possam ser ingeridos durante as 24 horas de realização do exame, conferindo agradável condição alimentar ao paciente, sem interferência na leitura do espectrofotômetro.

\section{MATERIAL E MÉTODO}

Foram testados 48 alimentos, com o probe de espectrofotometria, preparados e acondicionados no próprio recipiente de calibração.

O aparelho foi programado para registrar média de eventos a cada 16 segundos (possibilidade disponibilizada no "set up") e para ativar um canal de medição.

As frutas foram simplesmente batidas em liquidificador, o ovo mexido, os legumes e cereais, cozidos e depois levados ao triturador, sem adição de qualquer líquido. Os mingaus e a canjica foram preparados em dois volumes iguais de leite integral. Os pães, os queijos, o alho, a cebola e as carnes foram triturados em meio volume de água, e a margarina, os iogurtes e o leite foram testados in natura.

O probe era mergulhado em frasco de paredes escuras e tampa perfurada, permitindo sua passagem justa, capaz de bloquear entrada de luz, fornecido pelo fabricante do equipamento, contendo água para calibração; em seguida, colocado em outro frasco de características semelhantes contendo a amostra alimentar a ser testada durante cerca de 3 minutos, até que estabilizasse a leitura da absorbância; era lavado e calibrado para obtenção de nova medida de absorbância da amostra. O processo era ainda repetido mais uma vez.

Trabalhava-se, ao final, com a média das três medições efetuadas.

Adotou-se a densidade óptica da bilirrubina $(0,14)$ como divisor entre alimentos que poderiam ou não ser recomendados para compor a dieta durante a feitura da espectrofotometria do RGE.

\section{RESULTADOS}

Dos 48 elementos testados, um terço deles (Tabela 1) não se mostrou recomendável durante a realização da espectrofotometria, pois poderiam determinar resultados falso-positivos: ovo mexido, mingau de farinha láctea, feijão verde, beterraba, cenoura, chuchu, abobrinha, mandioquinha, milho verde cozido, laranja, caju, uva roxa, abacate, manga, mamão e pêssego.

Foi também possível observar com o método utilizado, de três medidas espectrofotométricas para cada elemento alimentar, a excelente reprodutibilidade das medições, muito semelhantes para cada alimento analisado. 
TABELA 1 - Absorbância dos alimentos testados pelo espectrofotômetro

\begin{tabular}{|c|c|c|c|c|c|}
\hline ALIMENTO & A1 & A2 & A3 & Amédia & Recom. \\
\hline leite integral & $\mathbf{0 , 0 1}$ & $\mathbf{0 , 0 1}$ & 0,01 & $\mathbf{0 , 0 1}$ & $\mathbf{S}$ \\
\hline pão de forma integral & $\mathbf{0 , 0 3}$ & $\mathbf{0 , 0 3}$ & $\mathbf{0 , 0 3}$ & $\mathbf{0 , 0 3}$ & $\mathbf{S}$ \\
\hline pão italiano & $\mathbf{0 , 0 2}$ & 0,04 & $\mathbf{0 , 0 2}$ & $\mathbf{0 , 0 3}$ & $\mathbf{S}$ \\
\hline margarina & $\mathbf{0 , 0 2}$ & $\mathbf{0 , 0 3}$ & $\mathbf{0 , 0 3}$ & $\mathbf{0 , 0 3}$ & $\mathbf{S}$ \\
\hline iogurte de morango & 0,06 & 0,04 & $\mathbf{0 , 0 7}$ & 0,05 & $\mathbf{S}$ \\
\hline iogurte natural & 0,04 & $\mathbf{0 , 0 2}$ & 0,01 & $\mathbf{0 , 0 2}$ & $\mathbf{S}$ \\
\hline queijo mozzarella & $\mathbf{0 , 0 1}$ & $\mathbf{0 , 0 1}$ & $\mathbf{0 , 0 1}$ & $\mathbf{0 , 0 1}$ & $\mathbf{S}$ \\
\hline queijo prato & $\mathbf{0 , 0 1}$ & $\mathbf{0 , 0 1}$ & 0,01 & $\mathbf{0 , 0 1}$ & $\mathbf{S}$ \\
\hline ovo mexido & 0,15 & 0,18 & 0,12 & 0,15 & $\mathrm{~N}$ \\
\hline mingau de aveia & 0,04 & 0,05 & 0,04 & 0,04 & $\mathbf{S}$ \\
\hline mingau de farinha láctea & 0,14 & 0,15 & 0,15 & 0,15 & $\mathrm{~N}$ \\
\hline mingau de maizena & $\mathbf{0 , 0 1}$ & $\mathbf{0 , 0 1}$ & $\mathbf{0 , 0 1}$ & $\mathbf{0 , 0 1}$ & $\mathbf{S}$ \\
\hline canjica & $\mathbf{0 , 0 1}$ & $\mathbf{0 , 0 1}$ & 0,01 & $\mathbf{0 , 0 1}$ & $\mathbf{S}$ \\
\hline arroz branco & $\mathbf{0 , 0 1}$ & $\mathbf{0 , 0 1}$ & $\mathbf{0 , 0 2}$ & $\mathbf{0 , 0 1}$ & $\mathbf{S}$ \\
\hline feijão verde & 0,18 & 0,22 & 0,21 & 0,20 & $\mathrm{~N}$ \\
\hline feijão branco & $\mathbf{0 , 1 0}$ & $\mathbf{0 , 1 2}$ & 0,09 & $\mathbf{0 , 1 0}$ & $\mathbf{S}$ \\
\hline espaguetti (molho de queijo branco) & 0,06 & $\mathbf{0 , 0 7}$ & $\mathbf{0 , 0 7}$ & $\mathbf{0 , 0 7}$ & $\mathbf{S}$ \\
\hline kani-kani (sem casca) & $\mathbf{0 , 0 1}$ & $\mathbf{0 , 0 2}$ & 0,01 & $\mathbf{0 , 0 1}$ & $\mathbf{S}$ \\
\hline carne branca de frango & $\mathbf{0 , 0 3}$ & $\mathbf{0 , 0 2}$ & $\mathbf{0 , 0 3}$ & $\mathbf{0 , 0 3}$ & $\mathbf{S}$ \\
\hline filé de peixe (frito em azeite) & 0,05 & 0,04 & 0,05 & 0,05 & $\mathbf{S}$ \\
\hline sardinha em lata (sem pele) & 0,09 & 0,09 & $\mathbf{0 , 1 0}$ & 0,09 & $\mathbf{S}$ \\
\hline ova de pescada & 0,09 & $\mathbf{0 , 1 0}$ & 0,11 & 0,10 & $\mathbf{S}$ \\
\hline alho & $\mathbf{0 , 0 3}$ & $\mathbf{0 , 0 1}$ & 0,05 & $\mathbf{0 , 0 3}$ & $\mathbf{S}$ \\
\hline cebola & $\mathbf{0 , 0 2}$ & $\mathbf{0 , 0 1}$ & 0,04 & $\mathbf{0 , 0 2}$ & $\mathbf{S}$ \\
\hline tomate ( sem casca) & $\mathbf{0 , 0 2}$ & 0,06 & $\mathbf{0 , 0 4}$ & $\mathbf{0 , 0 4}$ & $\mathbf{S}$ \\
\hline beterraba & 0,25 & 0,27 & 0,30 & 0,27 & $\mathrm{~N}$ \\
\hline cenoura & 0,21 & 0,20 & 0,21 & 0,20 & $\mathrm{~N}$ \\
\hline mandioca & 0,04 & 0,06 & 0,05 & 0,05 & $\mathbf{S}$ \\
\hline chuchu (sem casca) & 0,22 & 0,19 & 0,20 & 0,20 & $\mathrm{~N}$ \\
\hline abobrinha (sem casca) & 0,20 & 0,18 & 0,15 & 0,17 & $\mathrm{~N}$ \\
\hline champignon & $\mathbf{0 , 1 0}$ & 0,09 & 0,09 & 0,09 & $\mathbf{S}$ \\
\hline mandioquinha & 0,20 & 0,22 & 0,22 & 0,21 & $\mathrm{~N}$ \\
\hline milho verde cozido & 0,25 & 0,22 & 0,25 & 0,24 & $\mathrm{~N}$ \\
\hline palmito & 0,05 & $\mathbf{0 , 0 2}$ & 0,04 & $\mathbf{0 , 0 3}$ & $\mathbf{S}$ \\
\hline batata & 0,05 & 0,06 & 0,05 & $\mathbf{0 , 0 5}$ & $\mathbf{S}$ \\
\hline laranja & 0,32 & 0,32 & 0,33 & 0,32 & $\mathrm{~N}$ \\
\hline caju & 0,16 & 0,16 & 0,15 & 0,16 & $\mathrm{~N}$ \\
\hline uva branca (sem casca) & $\mathbf{0 , 1 0}$ & $\mathbf{0 , 1 0}$ & 0,09 & $\mathbf{0 , 1 0}$ & $\mathbf{S}$ \\
\hline $\operatorname{limão}$ & 0,09 & 0,09 & $\mathbf{0 , 1 0}$ & 0,09 & $\mathbf{S}$ \\
\hline uva roxa (sem casca) & 0,17 & 0,17 & 0,16 & 0,17 & $\mathrm{~N}$ \\
\hline abacate & 0,18 & 0,19 & 0,20 & 0,19 & $\mathrm{~N}$ \\
\hline banana & $\mathbf{0 , 0 3}$ & 0,04 & $\mathbf{0 , 0 3}$ & $\mathbf{0 , 0 3}$ & $\mathbf{S}$ \\
\hline manga & 0,69 & 0,70 & 0,69 & 0,70 & $\mathrm{~N}$ \\
\hline mamão & 0,70 & 0,70 & 0,71 & 0,70 & $\mathrm{~N}$ \\
\hline melão & $\mathbf{0 , 0 2}$ & $\mathbf{0 , 0 1}$ & 0,01 & $\mathbf{0 , 0 1}$ & $\mathbf{S}$ \\
\hline pêssego & 0,25 & 0,25 & 0,25 & 0,25 & $\mathrm{~N}$ \\
\hline pera d'água & 0,04 & $\mathbf{0 , 0 2}$ & $\mathbf{0 , 0 2}$ & $\mathbf{0 , 0 2}$ & $\mathbf{S}$ \\
\hline abacaxi & 0,05 & $\mathbf{0 , 0 3}$ & 0,05 & 0,04 & $\mathbf{S}$ \\
\hline
\end{tabular}

Os alimentos destacados em negrito têm absorbância média (Amédia) menor que a da bilirrubina $(0,14)$ e, portanto, são recomendáveis (Recom.) durante a realização do exame. 


\section{DISCUSSÃO}

Uma vez que a espectrofotometria tem demonstrado valor indiscutível no estudo da $\mathrm{DRGE}^{(1,4,6,7)}$, sua realização merece apuro técnico para que a exatidão do exame seja a maior possível.

O exame foi proposto recomendando-se (pelo próprio fabricante) "dieta branca" que não interferisse com a colorimetria.

Toma-se neste estudo a iniciativa de particularizar, originalmente, os alimentos que podem ser ingeridos sem alterar os resultados, oferecendo regime alimentar agradável ao paciente no período de análise espectrofotométrica.

A preparação dos alimentos para teste obedeceu a alguns critérios:

- a maioria faz parte da alimentação diária dos brasileiros;

- o cozimento e a trituração simulam o preparo habitual do alimento e boa mastigação;

- os mingaus seguiram a proporção de leite mais comumente utilizada;

- a preparação dos alimentos mais secos e/ou de maior consistência mimetiza o que aconteceria com a mastigação e mistura com a saliva;

- evitaram-se os de maior consistência ou maior conteúdo em fibras animais ou vegetais, pois este caráter, por si só, já os torna não recomendáveis, pela possibilidade de aderência ao probe, bloqueando sua leitura.

Bolachas e chás não foram testados, primeiro porque podem perfeitamente ser evitados no período de exame e também pela grande variabilidade de fabricação e preparo, respectivamente.

Também alguns alimentos de grande potencial oxidativo na presença de ácido clorídrico, como a maçã $\tilde{a}^{(3)}$, nem chegaram a ser testados, pois tem-se que contar com a possibilidade de deturpação do exame tanto pelo alimento em fluxo, como em refluxo, após atingir o estômago.
Esta preocupação é minimizada por estudo que verificou que alimentos que não alteram o exame em fluxo, também não o fazem em refluxo, desde que não sejam fortemente oxidáveis ${ }^{(9)}$.

Todos esses alimentos têm em comum o que se denomina na literatura de "cor forte" ${ }^{(8)}$, tons que incluem amarelo, vermelho e verde.

O probe mede a diferença de absorbância entre o comprimento de onda $470 \mathrm{~nm}$ (próximo ao comprimento de onda da bilirrubina: 453 $\mathrm{nm}$ ) e o comprimento de onda de referência (emitido pelo sistema), de $565 \mathrm{~nm}$, e a expressa em termos de "absorbância de bilirrubina" (escala de 0,0 a 1,0 unidade).

A calibração em água registra a linha de base, inferior a 0,01 unidade de absorbância e o próprio programa de análise do Bilitec $2000^{\circledR}$ estabelece 0,14 como divisor, acima do qual se registra refluxo biliar, isto é, presença de bilirrubina no refluxato, ou seja, presença de elemento cujo comprimento de onda é igual ou maior que o da bilirrubina.

A seleção "16" no aparelho registrador confere maior exatidão ao exame, pois valoriza-se a média de eventos que ocorrem durante 16 segundos (maior precisão do que selecionar 4 ou 8 , as outras opções do "set up").

Entende-se claramente, portanto, porque alimentos de "cor forte" representam grande risco de falso-positivo, pois se está trabalhando com colorimetria e quanto mais "escura" for a solução, maior o engano causado ao sistema de medição.

A "dieta clara" testada e recomendada por este estudo oferece excelentes opções de alimentação para os pacientes submetidos à espectrofotometria do refluxo, minimizando erros de medição e aumentando significativamente o cardápio recomendado pelo fabricante do sistema (Medtronics-Synectics).

Felix VN, Viebig RG. Recommended diet for reflux spectrophotometry. Arq Gastroenterol 2003;40(1):20-24.

ABSTRACT - Background - The spectrophotometric probe, which uses bilirubin as a marker for the detection of duodenoesophagic reflux is subject to interference from strongly colored foods, which can cause erroneously high bilirubin absorbance readings. To overcome this problem it is necessary to ingest a diet that is free from such substances. Objective - To test the absorbance of 48 different food substances in an in vitro environment. Material and method - Dry foods were blended with water or milk and non-dry solid foods were blended undiluted. It was utilized the proper calibration recipient to test them. Results - The absorbance of weakly colored foods was usually lesser than the commonly accepted threshold of 0.14 , and the absorbance of strongly colored foods was usually above this. Thirty-two from the 48 substances tested are suitable when the absorbance threshold is set at 0.14 , but scrambled eggs, lacteous flour mush, green beans, beetroot, carrot, chayote, squash, "baroa" potato, boiled corn, orange, cashew, purple grape, avocado, mango, papaya and peach can alter the results and must be avoided. Conclusion From the foods evaluated, enough are suitable at the 0.14 threshold to enable a suitable diet to be constructed for most patients during Bilitec studies.

HEADINGS - Duodenogastric reflux. Gastroesophageal reflux. Bile reflux. Esophagitis, peptic. Spectrophotometry. Diet. 


\section{REFERÊNCIAS BIBLIOGRÁFICAS}

1. Barrett MW, Myers JC, Watson DI. Detection of bile reflux: in vivo validation of the Bilitec Fibreoptic system. Dis Esophagus 2000;13:44-50.

2. Bechi P, Pucciani F, Baldini F. Long-term ambulatory enterogastric reflux monitoring. Validation of a new fibreoptic technique. Dig Dis Sci 1993;38:1297306.

3. deMann JM. Principles of food chemistry. 2 ed. New York: AVI Publishing; 1990.

4. DeMeester SR, DeMeester TR. Columnar mucosa and intestinal metaplasia of the esophagus: fifty years of controversy. Ann Surg 2000;231:303-21.

5. Felix VN, Viebig RG, editores. Atlas de eletromanometria - pHmetria - bilimetria. Guia prático de entendimento e indicação dos exames funcionais do esôfago. São Paulo: Cop L Print Formulários \& Editora Ltda; 2001.
6. Felix VN, Viebig RG. Spectrophotometry associated to pHmetry increase diagnostic accuracy of GERD. Hepatogastroenterology 2001;48 (Suppl 1):203.

7. Felix VN, Viebig RG. Twenty-four hours esophageal bilimetry: first Brazilian series. In: Pinotti HW, Cecconello I, Felix VN, Oliveira MA, editors. Recent advances in diseases of the esophagus. Bologna: Monduzzi; 2001. p. 25-30.

8. Kauer WKH, Peters JH, DeMeester TR, Ireland AP, Bremner CG, Hagen JA. Mixed reflux of gastric and duodenal juices is more harmful to the esophagus than gastric juice alone: the need for surgical therapy re-emphasized. Ann Surg 1995;222:525-33

9. Marshall REK, Angiasah A, Owen WA, Owen WJ. The relationship between acid and bile reflux and symptoms in gastro-oesophageal reflux disease. Gut 1997;40:182-7.

Recebido em $1 / 7 / 2002$ Aprovado em 22/10/2002. 